\title{
A review of non-destructive testing methods of composite materials
}

\begin{abstract}
A review provided about non-destructive testing (NDT) methods for the evaluation of composites. The review considers the capabilities of most common methods in composite NDT applications such as Visual Testing (VT or VI), Ultrasonic Testing (UT), Thermography, Radiographic Testing (RT), Electromagnetic Testing (ET), Acoustic Emission (AE), and Shearography Testing with respect to advantages and disadvantages of these methods. Then, methods categorized based on their intrinsic characteristics and their applications.
\end{abstract}

Keyword: NDT; Composite materials; Condition monitoring 